\title{
Is \% $\Delta$ SUVmax a Useful Indicator of Survival in Patients with Advanced Nonsmall-Cell Lung Cancer?
}

\author{
Angelina Cistaro, ${ }^{1,2,3}$ Natale Quartuccio, ${ }^{4}$ Alireza Mojtahedi, ${ }^{5}$ \\ Piercarlo Fania, ${ }^{1}$ Pier Luigi Filosso, ${ }^{6}$ Mariapaola Cucinotta, ${ }^{4}$ Alfredo Campennì, ${ }^{4}$ \\ Umberto Ficola, ${ }^{7}$ and Sergio Baldari ${ }^{4}$
}

${ }^{1}$ Positron Emission Tomography Centre, IRMET S.p.A., Euromedic, V.O. Vigliani 89, 10136 Turin, Italy

${ }^{2}$ PET Pediatric AIMN InterGroup, 10136 Turin, Italy

${ }^{3}$ Institute of Cognitive Sciences and Technologies, National Research Council, 00185 Rome, Italy

${ }^{4}$ Department of Biomedical Sciences and of Morphological and Functional Images, Nuclear Medicine Unit, University of Messina, 98125 Messina, Italy

${ }^{5}$ Nuclear Medicine Service, Memorial Sloan-Kettering Cancer Center, New York, NY 10021, USA

${ }^{6}$ Department of Thoracic Surgery, S. Giovanni Battista Hospital, 10126 Turin, Italy

${ }^{7}$ Department of Nuclear Medicine, La Maddalena Hospital, 90146 Palermo, Italy

Correspondence should be addressed to Angelina Cistaro; a.cistaro@irmet.com

Received 7 August 2013; Accepted 17 September 2013

Academic Editors: H. Abdel-Dayem, C.-H. Kao, and T. Smith

Copyright (C) 2013 Angelina Cistaro et al. This is an open access article distributed under the Creative Commons Attribution License, which permits unrestricted use, distribution, and reproduction in any medium, provided the original work is properly cited.

\begin{abstract}
Purpose. To investigate the impact of the maximum standardized uptake value (SUVmax), size of primary lung lesion, and $\% \triangle$ SUVmax on outcome (overall survival (OS) and 2-year disease-free survival (2-year DFS)) of patients with advanced nonsmallcell lung cancer (NSCLC). Materials and Methods. 86 stage III-IV NSCLC patients underwent 18 F-FDGPET/CT, before and after chemotherapy, and were classified into subgroups according to the response criteria of the European Organization for Research and Treatment of Cancer. SUVmax values and tumor size with the best prognostic significance were searched. Correlation between the SUVmax value and the initial response to therapy (best response) and the relationship between $\% \Delta S U V m a x$ and OS were assessed. Results. In patients in PD (20/86), the average pretreatment SUVmax was $11.8 \pm 5.23$, and the mean size of the primary lesion was $43.35 \mathrm{~mm} \pm 16.63$. In SD, PR, and CR patients (66/86), the average pretreatment SUVmax was $12.7 \pm 8.05$, and the mean size of the primary lesion was $41.6 \mathrm{~mm} \pm 21.15$. Correlation was identified only for $\% \Delta$ SUVmax; patients with PD $(\Delta$ SUVmax $>+25 \%)$ showed a worse OS than patients with $\triangle \mathrm{SUVmax}<+25 \%(\mathrm{CR}, \mathrm{PR}$, and SD) $(P=0.0235)$. Conclusions. In stage III-IV NSCLC, among the assessed factors, only $\% \Delta$ SUVmax may be considered as a useful prognostic factor.
\end{abstract}

\section{Introduction}

Among worldwide oncologic causes of death, lung cancer is the leading one [1]. Cross-sectional imaging using computed tomography (CT) combined with positron-emission tomography (PET) provides excellent information about anatomic and metabolic evaluation of malignancies. In order to stage, restage, and evaluate the treatment response in lung cancer, 2-deoxy-2-[18F]fluoro-D-glucose positron-emission tomography $\left({ }^{18} \mathrm{~F}-\mathrm{FDG}\right.$ PET/CT $)$ is widely used $[2,3]$. PET/CT has been utilized in the evaluation of response to treatment, and several studies demonstrated the correlation between decreased metabolic activity of the tumor following treatment and survival. Cisplatin-based chemotherapy is currently a key strand for treatment of advanced (stages III-IV) nonsmall-cell lung cancer (NSCLC). Despite the introduction of modern treatment protocols, prognosis of advanced NSCLC still remains poor, especially in comparison with disease in early stage [1]. While FDG-PET/CT has been demonstrated to provide additional prognostic information for patients with stages I-II NSCLC before treatment $[2,4]$, it has a limited prognostic role in stages III-IV [3]. However, 
TABLE 1: Characteristics of population.

\begin{tabular}{lc}
\hline Number of patients & 86 \\
Sex & $67 \mathrm{M}$ \\
Mean age & $19 \mathrm{~F}$ \\
Histotypes & $63.5 \mathrm{y}$ (range: $37-80)$ \\
$\quad$ Adenocarcinoma & 41 \\
Squamous & 16 \\
Large cell & 9 \\
Others & 20 \\
\hline
\end{tabular}

M: male; F: female; y: years.

previous study has demonstrated that FDG-PET/CT can be considered as a helpful tool in assessing therapeutic efficacy of chemotherapy in NSCLC [5]. According to the response criteria recommended by the European Organization for Research and Treatment of Cancer (EORTC), a metabolic response correlates with survival [6]. The early metabolic response, with significant reduction of tracer uptake compared with the baseline study before therapy, has been found predictive of favorable prognosis. Instead a slight reduction or an increased uptake is predictive of treatment failure or disease progression [5-7]. The aim of this study was to identify the possible cutoff values for SUVmax and size of primary lung lesion that have correlation with clinical outcome (OS and DFS) in patients with advanced (stages III-IV) NSCLC. We also evaluated the correlation between the pretreatment SUVmax and the initial response to therapy (best response) and between \% $\triangle$ SUVmax (the change between SUVmax before and after chemotherapy) and outcome.

\section{Materials and Methods}

Eighty-six patients $(67 \mathrm{M}$ and $19 \mathrm{~F} ; \mathrm{M} / \mathrm{F}$ ratio $=3.5: 1$; mean age $=63.5$ years; range $=37-80)$ were referred to IRMET Positron Emission Center S.p.A., Euromedic, in Turin, Italy, and Unit of Nuclear Medicine of La Maddalena Hospital in Palermo, Italy, for evaluation of their advanced non-small cell lung cancer. The histotypes were 41 (48\%) adenocarcinomas, $16(19 \%)$ squamous cell carcinomas, 9 (10\%) large cell carcinomas, and 20 (23\%) uncommon histotypes (others) (Table 1).

Patients were staged according to the TNM 7th edition [8] in stages IIIA, IIIB, and IV via information gathered through patient's chart including physical examination, routine blood test, bronchoscopy, contrast-enhanced computed tomography (CT) of the chest and upper abdomen, and brain CT. Survival and death information were retrieved by means of the hospitals databases. The research proposal was approved by Institutional Review Board and Ethics Committee. All patients underwent chemotherapeutic treatment based on cisplatin and taxanes drugs (135 mg of paclitaxel per square meter of body-surface area, administered over a 24-hour period on day 1 , followed by $75 \mathrm{mg}$ of cisplatin per square meter on day 2, every three weeks) and two FDG PET/CT whole body scan, one before (T0) and one after treatment (T1: one month). IIIA patients undergoing neoadjuvant chemotherapy and subsequent surgery were excluded from the study. Patients were excluded from the study also in case of (a) poor performance status; (b) Charlson Combined Age-Comorbidity Index $\geq 6$; (c) histological diagnosis of "bronchioloalveolar cell carcinoma" (BAC) subtype. PET/CT images were acquired at both centres (Turin and Palermo) on an integrated PET/CT scanner (Discovery ST, General Electric Medical System), 60 minutes after the injection of the $260-420 \mathrm{MBq}$ of ${ }^{18} \mathrm{~F}$-FDG, following 6 hours of fasting from the cranial base to the pelvis (total body) in 3D modality. The PET images were reconstructed iteratively on a $128 \times 128$ matrix. The SUVmax, within a spherical region of interest encompassing the entire volume, was calculated using a GE Xeleris workstation (General Electric Medical System, Milwaukee, WI, USA). The studies were interpreted by experienced nuclear medicine physician at both centers. The anatomical size of lung lesion was measured considering the maximum diameter of the lesion in the three planes [9]. Patients were followed up for 24 months with a frequency of clinical examination of every 3 months during the first year and every 6 months in the second year. A contrast-enhanced CT scan of the thorax was performed in all cases at 6 and 12 months, while the additional PET/CT scans were used in case of suspected morphological findings. At the end of the first-line chemotherapy treatment, the patients were further divided into the following 4 subgroups based on the $\% \triangle S U V m a x$ of the tumor, according to the EORTC response criteria [6]: stable disease (SD) $(\Delta<-25 \%$ to $\Delta<+25 \%)$, partial response $(\mathrm{PR})(\Delta>-25 \%)$, complete response (CR) (complete disappearance), and progressive disease (PD) $(\Delta>$ $+25 \%)$. Overall survival was calculated separately for PET responder patients $(\mathrm{SD}, \mathrm{PR}$, and $\mathrm{CR}$ ) and patients in disease progression (PD). Statistical correlation was searched for the following pairs of parameters: pretreatment SUVmax and outcome (OS and DFS), size and outcome (OS and DFS), pretreatment SUV max and best response, and size and best response using Student's $t$-test (the hypothesis was considered significant if $P \leq 0.05)$. Furthermore the correlation between $\% \Delta S U V \max$ and outcome (OS and DFS) was assessed using the chi-square test $\left(\chi^{2}\right.$-test).

\section{Results}

Thirteen patients out of 86 patients (15\%) were at stage IIIA, $18(21 \%)$ at stage IIIB, and $55(64 \%)$ at stage IV. At the staging, SUVmax and diameter of the primitive neoplastic lung lesion were evaluated in each patient. Patients showed an average baseline SUVmax of $12.5 \pm 7.43$ and a mean diameter of the primary lesion of $42 \pm 20 \mathrm{~mm}$. At the end of the first-line chemotherapy, according to EORTC criteria, 20/86 patients were in PD, 24/86 were SD, 35/86 had a PR, and 7/86 showed a CR (Figure 1).

Thirty-seven out of 86 patients were deceased at the follow-up time of 24 months (OS = 57\%). In the $20(23 \%)$ patients in progressive disease (PD), the recorded average pretreatment SUVmax was $11.8 \pm 5.23$, and the mean size of the primary lesion was $43.35 \mathrm{~mm} \pm 16.63$. Thirteen $/ 20$ patients had already died at the end of the followup (OS = $35 \%)$. In the $66(77 \%)$ patients in SD, PR, or CR, the average 

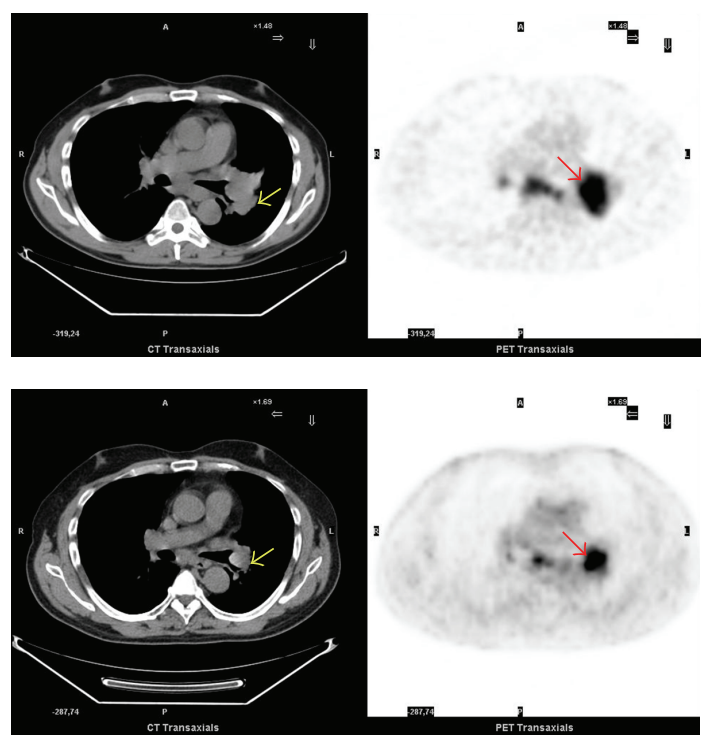

FIgUre 1: Pre- and posttreatment ${ }^{18}$ F-FDG PET/CT scan. Top: pretreatment ${ }^{18}$ F-FDG PET/CT axial image: staging on a patient with a left hilar adenocarcinoma (yellow arrow) with a SUVmax $=11$ (red arrow) and diameter of $40 \mathrm{~mm}$. Abnormal uptake present also in a subcarinal lymph node. Bottom: posttreatment ${ }^{18} \mathrm{~F}-\mathrm{FDG}$ PET/CT axial image: the study was performed one month later, at the end of the first-line chemotherapy. Note the decrease of the ${ }^{18} \mathrm{~F}$-FDG uptake (red arrow) in the primary lung lesion (SUVmax $=8)$ and also an important reduction of the lesion size (yellow arrow).

value of pretreatment SUVmax was $12.7 \pm 8.05$, and the mean size of the primary lesion was $41.6 \mathrm{~mm} \pm 21.15$ (Table 2).

24/66 patients passed away at the end of the followup (OS $=64 \%$ ). Using Student's $t$-test, no significant correlation was discovered between the following pairs of parameters: pretreatment SUVmax and outcome, and size and outcome, SUV max and best response, size and best response $(P=$ n.s.). Using the $\chi^{2}$-test, no SUVmax cutoff with prognostic significance was found ( $P=$ n.s.). However, analyzing the relationship between $\triangle S U V \max$ and overall survival, we found that patients with $\Delta<+25 \%$ (CR, PR, and SD) showed a better overall survival than patients in $\mathrm{PD}(\Delta>+25)(P=$ $0.0235, \chi^{2}$-test).

\section{Discussion}

The quantification of FDG uptake can be assessed by several parameters including standardized uptake value (SUV) or local metabolic rate of glucose. SUVmax still remains the most frequently used in clinical routine. In NSCLC, SUV quantification has been proved to have a role in the prediction of outcome [10-15]. For our group of 86 patients in stages IIIIV undergoing chemotherapy, no correlation neither between the pretreatment SUVmax in the primary tumor and survival (OS and DFS) nor between pretreatment SUVmax and best response has been found ( $P=$ n.s.). Even the attempt to apply the $\chi^{2}$-test to identify a SUVmax cut-off value with a powerful impact on the prognosis was unsuccessful. There are very few studies that investigated the SUVmax cut-off value
TABLE 2: Classes of metabolic response to therapy and average values of SUVmax and primitive lung lesion diameter.

\begin{tabular}{lccc}
\hline $\begin{array}{l}\text { Classes of } \\
\text { response to Number } \\
\text { therapy }\end{array}$ & $\begin{array}{c}\text { Average SUVmax } \\
\text { value }\end{array}$ & $\begin{array}{c}\text { Average primitive lung } \\
\text { lesion diameter }\end{array}$ \\
\hline CR & 66 & $12.7 \pm 8.05$ & $41.6 \mathrm{~mm} \pm 21.15$ \\
PR & 60 & $11.8 \pm 5.23$ & $43.35 \mathrm{~mm} \pm 16.63$ \\
SD & 20 & & \\
PD & &
\end{tabular}

and the prognosis of advanced nonsmall-cell lung cancer, and our data was very similar to those few reported in the literature $[3,16]$. Hoang and colleagues divided patients into two subgroups using a median SUV of 11.1 but did not find significant correlation between ${ }^{18}$ F-FDG uptake and median survival (16 months versus 12 months) [3]. The authors explained that the absence of correlation between survival and SUV of the primary tumor for patients in stages III-IV may be a consequence of the metastatic situation in these patients being more important for disease-free and overall survival. Other important variables are the clinical complexity of comorbidity of these patients, the prognostic score, and the side effects of the treatments $[17,18]$. In our study, the only parameter that showed a correlation with outcome (OS) was \% $\%$ SUVmax but was only marginally associated. Patients with $\Delta<+25 \%$ (CR, PR, and SD) showed a better overall survival than patients in $\operatorname{PD}(\Delta>+25)$ (64\% versus $35 \%)(P=0.0235)$. So far, in a single well-designed study on response assessment in advanced stages (stage IIIB or IV), Weber et al. [5] showed that a reduction of metabolic activity already after 1 cycle of chemotherapy is closely correlated with final outcome of therapy. Fifty-seven patients scheduled to receive platinum-based chemotherapy underwent PET scan before and after the first cycle of therapy. A decrease in SUV of $20 \%$ or more after 1 cycle of chemotherapy was associated with a longer time to progression (163 versus $54 \mathrm{~d}$ ) and longer median OS time ( 252 versus $151 \mathrm{~d})$. The authors showed also a significantly higher 1-year survival rate in metabolic responders compared with nonresponders (44\% versus $10 \%$ ) [5]. Similarly, Kim and colleagues described the prognostic usefulness of ${ }^{18}$ F-FDG PET after chemotherapy in patients with advanced stages III and IV NSCLC and demonstrated that when the tumor reveals more than $17.85 \%$ reduction of $\% \Delta S U V m a x$ the survival could be predicted, but their study was limited at only 19 patients [19]. Further confirmatory data come from the study performed by Mac Manus et al. [20] showing a significantly longer median survival for patients with complete metabolic response than for patients with incomplete metabolic response (31 versus 11 months).

Our study had several limitations including lack of SUV assessment in each single histotype and other more recent metabolic indices, such the Metabolic Tumor Volume.

\section{Conclusions}

Our data suggested that SUVmax has not prognostic predictive value in the advanced NSCLC (III-IV). No useful 
correlation between SUVmax, tumor size, and clinical outcome of NSCLC patients in stages III-IV was found. In patients with advanced disease, only $\% \Delta$ SUVmax may be considered as a useful prognostic factor. Additional studies are required to further accurately assess the role of SUVmax in advanced nonsmall-cell lung cancer.

\section{Conflict of Interests}

The authors report no conflict of interests in this work.

\section{References}

[1] P. Saintigny and J. A. Burger, "Recent advances in non-small cell lung cancer biology and clinical management," Discovery Medicine, vol. 13, pp. 287-297, 2012.

[2] R. J. Downey, T. Akhurst, M. Gonen et al., "Preoperative F18 fluorodeoxyglucose-positron emission tomography maximal standardized uptake value predicts survival after lung cancer resection," Journal of Clinical Oncology, vol. 22, no. 16, pp. 32553260, 2004.

[3] J. K. Hoang, L. F. Hoagland, R. E. Coleman, A. D. Coan, J. E. Herndon II, and E. F. Patz Jr., "Prognostic value of fluorine-18 fluorodeoxyglucose positron emission tomography imaging in patients with advanced-stage non-small-cell lung carcinoma," Journal of Clinical Oncology, vol. 26, no. 9, pp. 1459-1464, 2008.

[4] A. Cistaro, N. Quartuccio, A. Mojtahedi et al., "Prediction of 2 years-survival in patients with stage I and II non-small cell lung cancer utilizing 18F-FDG PET/CT SUV quantification," Radiology and Oncology, vol. 47, no. 3, pp. 219-223, 2013.

[5] W. A. Weber, V. Petersen, B. Schmidt et al., "Positron emission tomography in non-small-cell lung cancer: prediction of response to chemotherapy by quantitative assessment of glucose use," Journal of Clinical Oncology, vol. 21, no. 14, pp. 2651-2657, 2003.

[6] H. Young, R. Baum, U. Cremerius et al., "Measurement of clinical and subclinical tumour response using $\left[{ }^{18} \mathrm{~F}\right]$-fluorodeoxyglucose and positron emission tomography: review and 1999 EORTC recommendations," European Journal of Cancer, vol. 35, no. 13, pp. 1773-1782, 1999.

[7] W. Huang, T. Zhou, L. Ma et al., "Standard uptake value and metabolic tumor volume of 18 F-FDG PET/CT predict shortterm outcome early in the course of chemoradiotherapy in advanced non-small cell lung cancer," European Journal of Nuclear Medicine and Molecular Imaging, vol. 38, no. 9, pp. 1628-1635, 2011.

[8] American Joint Committee on Cancer, AJCC Cancer Staging Manual, Springer, New York, NY, USA, 7th edition, 2010.

[9] E. A. Eisenhauer, P. Therasse, J. Bogaerts et al., "New response evaluation criteria in solid tumours: revised RECIST guideline (version 1.1)," European Journal of Cancer, vol. 45, no. 2, pp. 228247, 2009.

[10] W. A. Weber, S. I. Ziegler, R. Thödtmann, A.-R. Hanauske, and M. Schwaiger, "Reproducibility of metabolic measurements in malignant tumors using FDG PET," Journal of Nuclear Medicine, vol. 40, no. 11, pp. 1771-1777, 1999.

[11] S. C. Huang, "Anatomy of SUV. Standardized uptake value," Nuclear Medicine and Biology, vol. 27, pp. 643-646, 2000.

[12] M. C. Adams, T. G. Turkington, J. M. Wilson, and T. Z. Wong, "A systematic review of the factors affecting accuracy of SUV measurements," American Journal of Roentgenology, vol. 195, no. 2, pp. 310-320, 2010.

[13] R. Boellaard, M. J. O’Doherty, W. A. Weber et al., "FDG PET and PET/CT: EANM procedure guidelines for tumour PET imaging: version 1.0," European Journal of Nuclear Medicine and Molecular Imaging, vol. 37, no. 1, pp. 181-200, 2010.

[14] R. J. Cerfolio, A. S. Bryant, B. Ohja, and A. A. Bartolucci, "The maximum standardized uptake values on positron emission tomography of a non-small cell lung cancer predict stage, recurrence, and survival," Journal of Thoracic and Cardiovascular Surgery, vol. 130, no. 1, pp. 151-159, 2005.

[15] A. van Baardwijk, C. Dooms, R. J. van Suylen et al., “The maximum uptake of $18 \mathrm{~F}$-deoxyglucose on positron emission tomography scan correlates with survival, hypoxia inducible factor- $1 \alpha$ and GLUT-1 in non-small cell lung cancer," European Journal of Cancer, vol. 43, no. 9, pp. 1392-1398, 2007.

[16] H. Vesselle, J. D. Freeman, L. Wiens et al., "Fluorodeoxyglucose uptake of primary non-small cell lung cancer at positron emission tomography: new contrary data on prognostic role," Clinical Cancer Research, vol. 13, no. 11, pp. 3255-3263, 2007.

[17] N. Al-Sarraf, K. Gately, J. Lucey et al., "Clinical implication and prognostic significance of standardised uptake value of primary non-small cell lung cancer on positron emission tomography: analysis of 176 cases," European Journal of Cardio-thoracic Surgery, vol. 34, no. 4, pp. 892-897, 2008.

[18] C. T. Li, M. Marek, S. Z. Guclu et al., "Smoking and prognostic factors in an observational setting in patients with advanced non-small cell lung carcinoma," Journal of Cancer, vol. 2, pp. 52 61, 2011.

[19] Y. S. Kim, M. K. Lee, S. J. Kim et al., "Prognostic stratification using F-18 FDG PET/CT in patients with advanced stage (Stage III and IV) non-small cell lung cancer," Neoplasma, vol. 57, no. 3, pp. 241-246, 2010.

[20] M. P. Mac Manus, R. J. Hicks, J. P. Matthews, A. Wirth, D. Rischin, and D. L. Ball, "Metabolic (FDG-PET) response after radical radiotherapy/chemoradiotherapy for non-small cell lung cancer correlates with patterns of failure," Lung Cancer, vol. 49, no. 1, pp. 95-108, 2005. 


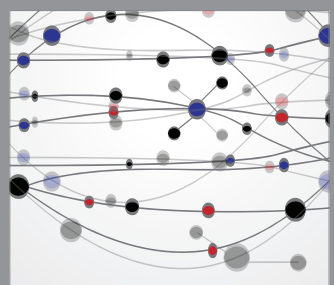

The Scientific World Journal
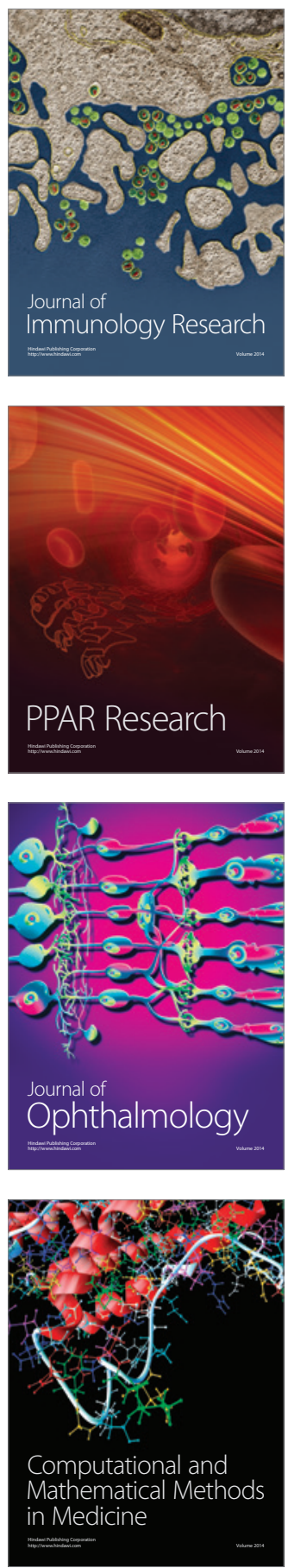

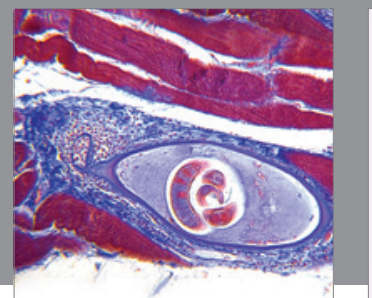

Gastroenterology

Research and Practice
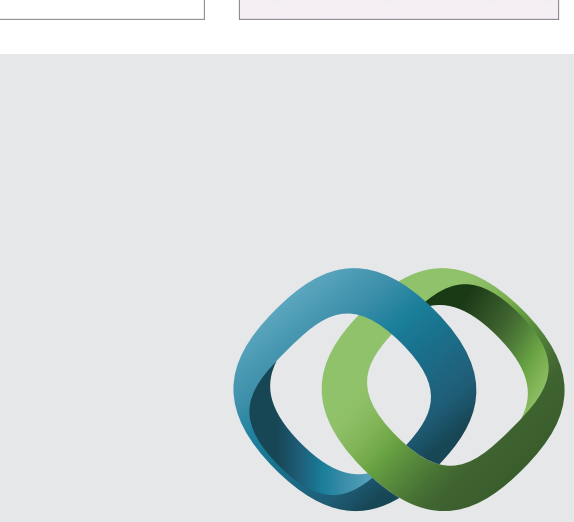

\section{Hindawi}

Submit your manuscripts at

http://www.hindawi.com
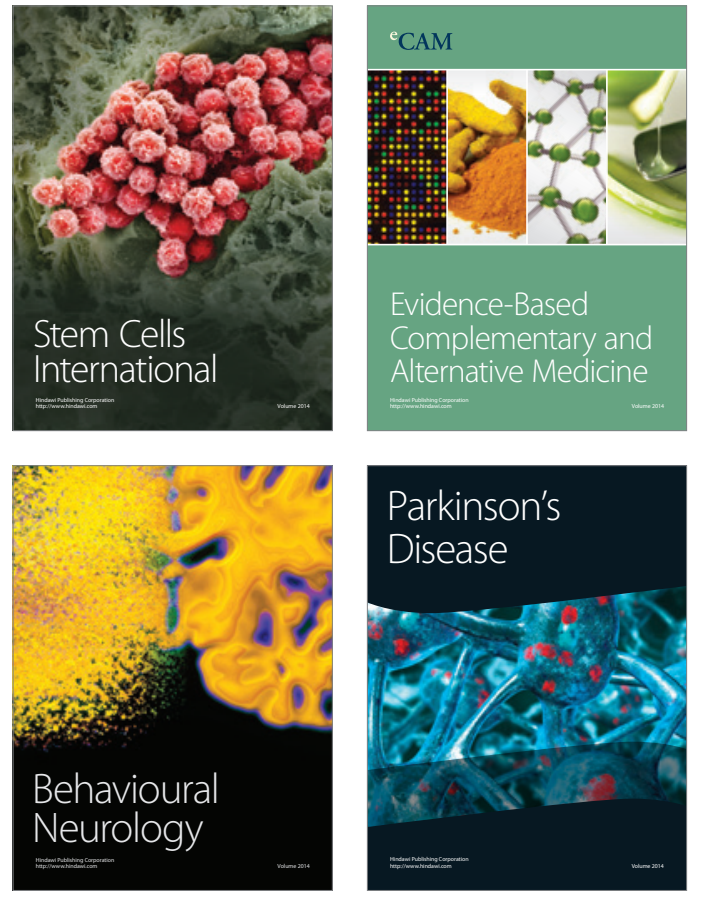
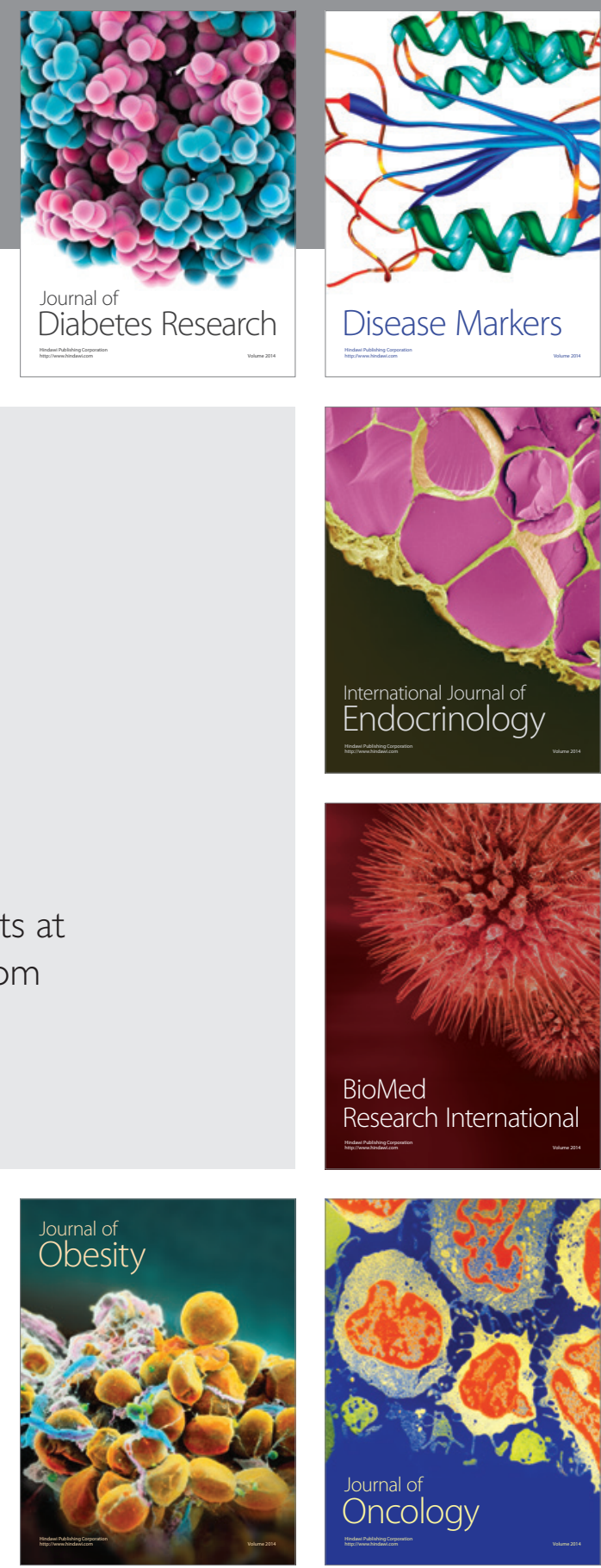

Disease Markers
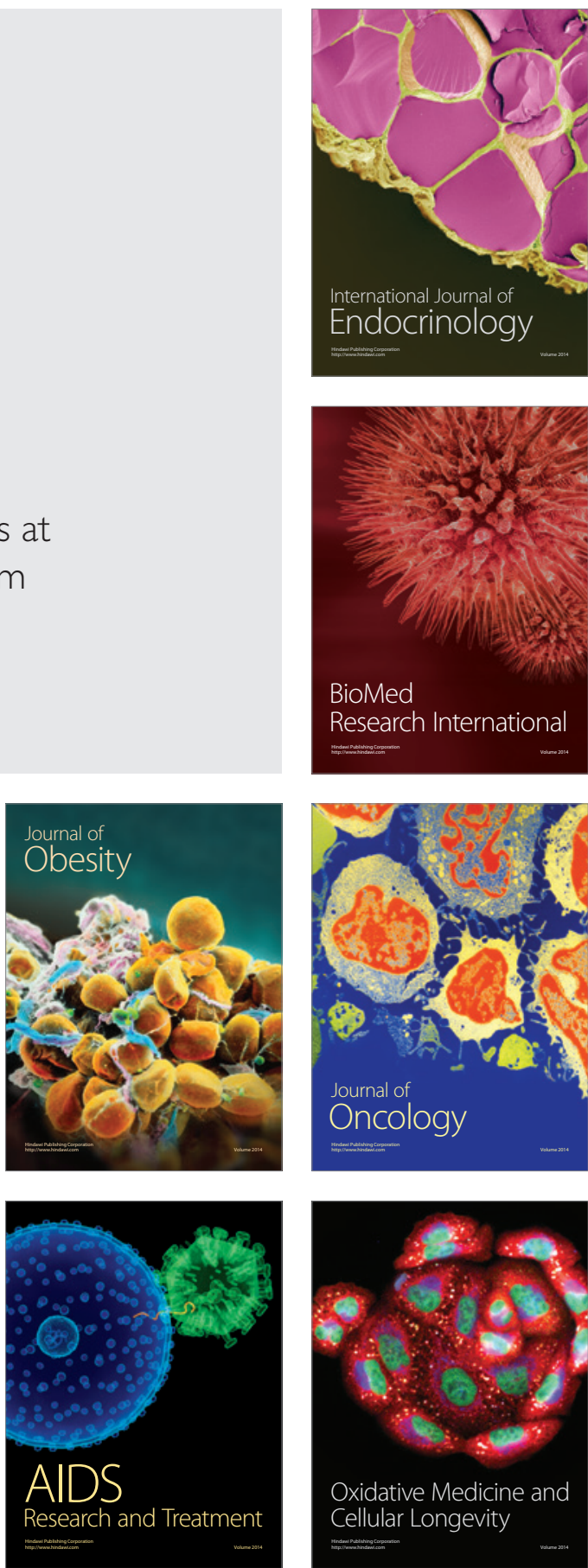